\title{
Aberration Correction and Electron Holography
}

\author{
Hannes Lichte, Dorin Geiger, Martin Linck and Michael Lehmann*
}

Triebenberg Laboratory, Institute of Structure Physics, Technische Universitaet Dresden, Germany, www.triebenberg.de

* Institut für Optik und Atomare Physik, Technische Universitaet Berlin, Berlin, Germany, www.ioap.tu-berlin.de/menue/arbeitsgruppen/ag_lehmann

Otto Scherzer has shown that aberrations cannot be avoided with the usual round electron lenses [1]. However, he also has shown how electron microscopy can make the best out of it. In his famous paper [2] he outlined the wave optical aspects in a TEM and derived the transfer functions for both Phase Contrast and Amplitude Contrast. He showed that aberrated imaging of atoms - considered as phase objects - needs optimizing phase contrast by means of the so-called Scherzer-focus. At the end, after improving the performance of electron sources and lenses, atomic structures were visible, however, averaged about the point-spread function ("delocalization") with a diameter of about five times Scherzer resolution. Despite the remaining restrictions, TEM contributed indispensably to the progress in materials and life sciences.

Amongst others, since 1936 Scherzer struggled for an aberration corrector to overcome the resolution limit with the help of non-round electron lenses. His scholars Harald Rose [3] and Maximilian Haider [4] finally solved the involved problems and built the aberration corrector hence brought TEM to real atomic resolution; a lateral resolution of $0.05 \mathrm{~nm}$ is within reach. With these correctors, the point-spread function provoked by the residual aberrations is finally smaller than the atoms; therefore, position and intensity of atoms can reliably be interpreted. Additionally, like in any optical system, opening up the imaging aperture improves not only lateral resolution but also enhances the signal strength by increasing the collection efficiency for the diffracted electron waves. Therefore, the perceptibility of weak structures such as light atoms is considerably improved.

Nevertheless, already from the papers of Scherzer, it became also evident that there is an additional problem: the object modulates both amplitude and phase of the electron waves. Therefore, the question remains What do we see in the intensity image? With correction of aberrations, these questions get even more severe in that phase modulations are completely invisible in an intensity image recorded at perfect correction. A solution is offered by electron holography.

Dennis Gabor proposed electron holography as a lens-less imaging method in order to by-pass the limitations presented by the aberrations [5]. In fact, lens-less electron holography is not yet successful because of the comparably poor coherence provided by electron beams. Therefore lenses are needed to take image plane holograms at highest lateral resolution; fortunately, the effects of lens aberrations in the reconstructed wave can be corrected a-posteriori; a review is found in [6]. From such a hologram, the complete object exit wave can uniquely be determined in an amplitude image and a phase image, and hence all information needed for a complete interpretation in terms of the object is available. Then, phase images allow revealing details of the atomic structure, such as

- difference of atomic numbers of different constituents

- number of atoms in an atomic column

- interatomic electric potentials

- potentials across interfaces

Examples are given in [7]. 
Performance Limits

The performance of electron holography mainly depends on the TEM used for recording the hologram. The optimal reachable lateral resolution is given by the information limit of the microscope. We have reached about $0.1 \mathrm{~nm}$ so far using a Philips CM30 TEM. Besides lateral resolution, the signal resolution, i.e. the discernibility of details of the phase image, is often more essential to see the very weak object details. The two aspects, i.e. lateral and signal resolution, can be combined in one figure of merit, which we call the Information Content InfoCont $=n_{\varphi} n_{\text {rec }}$ [8]. It is the product of the number $n_{\varphi}$ of phase values distinguishable in the range $(0,2 \pi)$, and the number $n_{\text {rec }}$ of pixels reconstructed across the field of view. It turns out that InfoCont depends on the brightness of the electron emitter, stability of the TEM, and the quality of the electron detector used. In our case, InfoCont $=7100$ is achievable. This means for example that, to see details of $2 \pi / 50$ such as fields of single atoms, corresponding to $n_{\varphi}=50$, the number of reconstructed pixels is limited to $n_{\text {rec }}=140$. Reachable lateral resolution and field of view are related accordingly: aiming at a lateral resolution of $0.1 \mathrm{~nm}$, the reachable field of view is limited to $7 \mathrm{~nm}$, because one resolution element is made up by two pixels (Nyquist sampling theorem).

Electron holography using an aberration corrected TEM

The coherent aberrations of the TEM can be corrected for a-posteriori during reconstruction of the electron wave. Therefore, at first glance, aberrations do not play a decisive role. However, they do for signal resolution, because each point in the image wave is smeared out by the comparably large point-spread function, even at optimum focus for holography. Therefore, an accordingly wide hologram has to be recorded and hence the coherent current density drops. This gives rise to considerable quantum noise. With an aberration corrector, the quality of holograms is much improved in that the noise level is decreased. In our Tecnai F20-Cs corr TEM, together with the signal enhancement, the signal/noise properties are found improved by a factor $\approx 4$ [9]. Furthermore, the capabilities of a-posteriori correction are very favorable for a-posteriori fine-tuning of aberrations in the reconstructed wave. The performance, essentially restricted by quantum noise, allows atomic resolution both laterally and in signal. Any improvement of electron microscopy, such as of lateral resolution and of brightness of the electron gun, will enhance the capabilities also of electron holography. Presently, the most severe challenge is to understand and distinguish the phase shifts arising at atomic dimensions, such as from atoms, fields, boundaries and defects, as well as from mistilt and thickness variations of the object.

[1] O. Scherzer, Z. Physik 101(1936), 593

[2] O. Scherzer, J. Appl. Phys. 20(1949), 20

[3] H. Rose, Optik 85(1990), 19

[4] M. Haider, Rose H, Uhlemann S, Schwan E, Kabius B, and Urban K, J. Electron Microscopy 47(1998), 395

[5] D. Gabor, Nature 161(1948), 563

[6] H. Lichte and M. Lehmann, Rep. Prog. Phys. 71(2008), 016102.

[7] H. Lichte, P. Formánek, A. Lenk, M. Linck, Ch. Matzeck, M. Lehmann and P. Simon, Annual Review of Materials Research 37(2007), 539

[8] H. Lichte, Ultramicroscopy 108(2008), 256

[9] D. Geiger, H. Lichte, M. Linck, M. Lehmann, Microsc. Microanal. 14, 68-81, 2008

[10] These projects are financially supported by the Deutsche Forschungsgemeinschaft and by the European Union (Framework 6 Integrated Infrastructure, Reference 026019 ESTEEM). 